\title{
A comparison between different anti-retroviral therapy regimes on soluble inflammation markers: a pilot study
}

\author{
Martina Maritati ${ }^{*} \mathbb{C}$, Trentini Alessandro ${ }^{2}$, Nunzia Zanotta ${ }^{3}$, Manola Comar $^{4}$, Tiziana Bellini $^{2}$, Laura Sighinolf ${ }^{5}$ \\ and Carlo Contini ${ }^{1}$
}

\begin{abstract}
Background: Although HIV-related deaths have decreased dramatically following the introduction of antiretroviral therapy (ART), HIV infection itself causes increased morbidity and mortality for both non-AIDS-related events or chronic inflammation and immune activation. The use of certain antiretroviral drugs can contribute to this process.

Methods: We investigated 26 potential biomarkers in serum samples from HIV-1 infected patients virologically suppressed under ART. The main objective of our study was to evaluate if virological suppression achieved with a triple drug regimen containing tenofovir disoproxil fumarate co-formulated with emtricitabine (TDF/FTC) as backbone, could correlate with a better immunological and inflammatory profile in relation to the third class of antiretroviral drug administered. The eligible patients were then divided into 3 groups in relation to the third drug associated with TDF/FTC: nucleoside reverse transcriptase inhibitors (NNRTI) (Group 1, $n=16$ ), protease inhibitors (PI) (Group 2, $n=17$ ) and integrase inhibitors (INI) (Group 3, $\mathrm{n}=16$ ).

Results: Inflammatory cytokines and chemokines were more represented in Group 2 than in Group 3 (IL-1Ra, $p=0.013 ; \mathrm{IL}-12 p 70 p=0.039 ;$ TNF- $a p=0.041 ; \mathrm{IL}-8, p=0.027 ; \mathrm{MIP1} \beta, p=0.033$ ). Eotaxin showed lower levels in Group 1 compared to Group $2(p=0.010)$, while IP-10 was significantly lower in Group 1 compared to both Group 2 and Group 3 ( $p=0.003$ and $p=0.007$, respectively).

Conclusions: Our results seem to discourage the administration of PI as a third drug in a virologically effective antiretroviral regimen, as its use is linked to the detection of higher levels of pro-inflammatory mediators in comparison with INI and NNRTI.
\end{abstract}

Keywords: HIV, Virological suppression, Antiretroviral therapy, Immune activation, Chronic inflammation

\section{Background}

The introduction of antiretroviral therapy (ART) has contributed to the reduction of AIDS-related mortality among HIV-positive patients [1] and to the induced chronicity of HIV infection. Triple drug regimens that include two nucleos(t)ide reverse transcriptase inhibitors (NRTI)

*Correspondence: mrtmtn@unife.it

${ }^{1}$ Section of Infectious Diseases, Department of Medical Sciences, University of Ferrara, 44124 Ferrara, Italy

Full list of author information is available at the end of the article as the backbone plus a base agent of another class, protease inhibitors (PI), integrase inhibitors (INI) or nucleoside reverse transcriptase inhibitors (NNRTI) [2], have been shown to control viral replication [3] and represent the gold standard for the treatment of HIV infection in both antiretroviral-naïve and antiretroviral experienced patients. Now, these patients live longer, although with a higher prevalence of non-infectious comorbidities including cardiovascular disease, diabetes, renal dysfunction and liver damage. Although these comorbidities are quite common among the general population [4], they 
are increasingly frequent in patients with ART [5] more likely due to the inflammatory process related to HIV and the adverse pharmacological effects of ART [6-11].

In this regard, the international guidelines on the management of HIV-positive patients recommend a change in lifestyle (smoking cessation, changes in diet and physical activity) and, in case of high LDL and/or hypertriglyceridemia values, the administration of statins. In addition to these measures, it is highly recommended to move to an antiretroviral regimen that limits the impact on lipid metabolism, suggesting the adoption of a therapy "tailored" to the needs of the patient.

Clinical studies have also shown that the transition from therapies such as PI or NNRTI (efavirenz) to integrase inhibitors (INI) (e.g. raltegravir, dolutegravir) leads to an improvement in the lipid profile, inflammatory pattern, and chronic immune activation. In fact, HIV infection combined with ART is directly associated with immune activation [12, 13], even in virologically suppressed patients. It is known, indeed, that the dysregulation of inflammatory processes induced by HIV occurs through multiple pathways [14], including microbial translocation [15].

To better understand this phenomenon and to optimize therapeutic management, some authors have recently been stimulated to seek and validate new immunological and inflammatory predictive biomarkers.

So far, most studies examining changes in levels of inflammatory biomarkers in HIV-infected individuals have been limited by small study populations, crossdrawings and/or a small number of biomarkers [16]. In this regard, recent technical advances, including the development of multiplexed cytokine tests, have contributed to a more efficient measurement of multiple inflammatory biomarkers [17].

In this study, we investigated 26 potential biomarkers, linked to systemic inflammation, immune activation and senescence in HIV patients under ART for at least one year and virologically suppressed (HIV-RNA $<20$ copies $/ \mathrm{ml}$ ) for at least 6 months. All the enrolled patients were treated with an ART triple drug regimen containing tenofovir disoproxil fumarate co-formulated with emtricitabine (TDF/FTC) as backbone. The main aim of our study was to assess whether virological suppression can be related to a better immunological and inflammatory profile, in relation to the third antiretroviral drug administered.

\section{Methods}

\section{Study patients and design}

Forty-nine caucasian HIV-1 positive patients aged $\geq 40$ years, who have taken ART for at least one year, were enrolled at the Infectious Diseases Department
(HIV Section) University-Hospital of Ferrara. Strict inclusion criteria were considered: (1) virological suppression for at least 6 months obtained with a triple drug regimen containing TDF/FTC as a backbone; (2) Cardiovascular risk $(\mathrm{CVR}) \geq 7.5 \%$, calculated according to the Atherosclerotic Cardiovascular Disease (ASCVD) algorithm of American College of Cardiology/American Heart Association (ACC-AHA) [18], in the absence of a regimen with statin and / or aspirin. The presence of comorbidities (chronic inflammatory diseases, neoplasms, diabetes, obesity, etc.) and co-infections represented a strict exclusion criterion.

Eligible patients were then divided into groups according to the third drug associated with TDF/FTC at the time of enrollment: NNRTI (efavirenz) (Group 1, $\mathrm{n}=16$ ), PI (atazanavir/r or darunavir/r) (Group $2, \mathrm{n}=17$ ) and INI (raltegravir) (Group 3, $\mathrm{n}=16$ ).

This study is in line with the Code of Ethics of the World Medical Association (Declaration of Helsinki) and was conducted according to the guidelines for Good Clinical Practice (European Medicines Agency). The study was approved by The Local Ethic Committee and written informed consent was obtained from each patient prior to inclusion in the study.

\section{Serum sample collection}

Serum samples were obtained from the 49 enrolled subjects. The whole blood of each subject was collected in a covered tube without anticoagulants and left to clot undisturbed at room temperature for $20 \mathrm{~min}$. The clot was then removed by centrifuging at $1500 \times \mathrm{g}$ for $10 \mathrm{~min}$ in a refrigerated centrifuge. After centrifugation, the serum was immediately transferred to a clean polypropylene tube. All sera were maintained at $-80{ }^{\circ} \mathrm{C}$ until cytokines analysis.

\section{Chemokines and cytokines analysis}

The main outcome measures were the quantification of cytokine concentrations and growth factors in biological samples based on magnetic bead multiplex immunoassays (Bio-Plex, BIO-RAD Laboratories, Milano, Italy). Luminex multiplex panel technology was used for simultaneous measurement of a panel of 26 analytes including cytokines, chemokines and growth factors (IL-1, IL-2, IL-1ra, IL-4, IL-5, IL-6, IL-9, IL-10, IL-12p70, IL-13, IL-15, TNF- $\alpha$, IL-17, IL-18, IFN- $\gamma$, MIP1 $\alpha$, MIP1 $\beta$, IL-8, IP-10, RANTES, MCP-1, GM-CSF, G-CSF, IL-7, VEGF, PDGF-bb) according to the method of Comar et al. 2014. Briefly, $50 \mu \mathrm{l}$ of diluted serum samples (1:4) and reaction standards were added, in duplicate, to a 96 multiwells plate containing analyte beads followed by incubation for $30 \mathrm{~min}$ at room temperature. After washing, the antibody-biotin reporter was added and incubated for $10 \mathrm{~min}$ 
with streptavidin-phycoerythrin. Cytokines levels were determined using the Bio-Plex array reader (Luminex, Austin, TX). The Bio-Plex Manager software automatically optimized the standard curves and returned the reading data as Median Fluorescence Intensity (MFI) and concentration $(\mathrm{pg} / \mathrm{mL}$ ). An ELISA set (Quantikine ELISA-Human CCL5/Rantes immunoassay, RnD system, Minneapolis, MN) with a mean minimum detectable dose of $2.0 \mathrm{pg} / \mathrm{ml}$ was used as confirmatory test according to manufacturer's instruction [17].

\section{Statistical analysis}

The normality of distribution of continuous variables was assessed by Kolmogorov-Smirnov test; since variables were not normally distributed, group comparisons were made by Kruskall-Wallis followed by the Mann-Whitney $U$ test corrected for multiple comparisons (Bonferroni). To correct for possible confounding factors, such as age, sex, smoke and disease duration, group comparisons were performed on natural logarithm-transformed variables by ANCOVA, including the variables listed as covariates. The Spearman's Rank Test was used to analyze bivariate correlations. The categorical variables were compared by Chi Square Test.

Data analysis was performed using SPSS Statistics for Windows, version 21.0 (SPSS, Inc., Chicago, IL, USA). Two-tailed probability values $<0.05$ were considered statistically significant.

\section{Results}

The demographic and clinical characteristics of the population studied are summarized in Table 1.

\section{Table 1 Demographic and clinical characteristic} of the study population

\begin{tabular}{lccc}
\hline & Group 1 & Group 2 & Group 3 \\
\hline Age (years) & $49.7(45.9-$ & $52.9(47.4-$ & 53.7 \\
& $57.0)$ & $57.1)$ & $(45.3-$ \\
& & & $61.6)$ \\
CD4+ & $857(669-$ & 727 & $701(545-$ \\
& $1045)$ & $(527-926)$ & $857)$ \\
Sex (female, \%) & 44.4 & 50.0 & 33.3 \\
Smoking status (smokers, \%) & 55.6 & 50.0 & 50.0 \\
Disease Duration (years) & $16.0(11.3-$ & $15.5(11.6-$ & $10.4(8.7-$ \\
& $20.7)$ & $19.5)$ & $12.2)$ \\
Triglycerides (mg/dL) & $150(83-217)$ & $134(91-176)$ & $126(103-$ \\
& & & $149)$ \\
Total cholesterol (mg/dL) & 220 & 210 & $229(210-$ \\
& $(193-247)$ & $(185-235)$ & $248)$
\end{tabular}

Continue variables are expressed as mean (95\% Confidence Interval). Categorical variables are expressed as percentage
Of the 49 patients enrolled, 14 were women $(28.57 \%)$ and 35 were males $(71.43 \%)$.

The values of triglycerides were high $(>150 \mathrm{mg} / \mathrm{dL})$ in $33 \%, 35 \%$ and $31 \%$ of patients from NNRTI, PI and INI group, respectively. The frequencies were not different between the three groups (chi-square $(2)=0.061$, $p=0.970$ ).

Total cholesterol was high (>200 mg/dL) in 60\%, 53\% and $69 \%$ of patients from NNRTI, PI and INI group, respectively. Also in this case, the frequencies of high total cholesterol levels were not different between the three groups (chi-square $(2)=0.863, p=0.650$ ).

A first exploratory statistical analysis showed that one patient, belonging to Group 1, showed extreme values (absolute value of $\mathrm{z}$-score $>1.6)$ for $8(30.7 \%)$ of the 26 cytokines analyzed and, consequently, was excluded from the analysis.

Although statistical significance was achieved for only few mediators, probably because of the small sample size, our results showed that 7 of the 26 immune mediators (18.2\%), analyzed between the different groups, are influenced by the type of third drug administered (Additional file 1. Figure S1).

As for the group of innate immunity mediators, some traditionally inflammatory cytokines and chemokines were more represented in PI-treated serum samples with higher levels than those treated with INI (IL-1Ra, $\mathrm{p}=0.013 ; \mathrm{IL}-12 \mathrm{p} 70 \mathrm{p}=0.039 ; \mathrm{TNF}-\alpha p=0.041 ; \mathrm{IL}-8$, $p=0.027$; MIP1 $\beta, p=0.033)$. Eotaxin showed lower levels in Group 1 compared to Group $2(\mathrm{p}=0.010)$, while IP-10 was significantly lower in Group 1 compared to both Group 2 and Group $3(p=0.003$ and $p=0.007$, respectively). Of note, almost $47 \%$ of patients in both NNRTI and PI groups had abnormal cytokine values ( $\mid z$-score $\mid>1.6)$, whereas $25 \%$ in the INI group showed this behavior (Additional file 1. Table S2). However, the difference in frequencies between groups did not reach statistical significance (Chi-square $(2)=2.135, P=0.344$ ).

As shown in Table 2, statistically significant correlations between disease duration and levels of some immune mediators were found in the overall population.

The univariate analysis (ANOVA) showed that there were no statistical differences in disease duration between groups, although INI patients showed a tendency to have lower disease duration values. In order to correct the cytokine values for possible confounding factors (age, sex, smoking status, duration of disease), we used an ANCOVA approach on natural logarithmtransformed variables. As summarized in Additional file 1. Table S1 and Fig. 1, some of the immune mediators tested were affected by confounding factors, in particular IL-1ra, IL12p70 and TNF- $\alpha$ which resulted no longer 


\begin{tabular}{lcc}
$\begin{array}{l}\text { Table } 2 \\
\text { between Interleukins levels and HIV diseases duration }\end{array}$ \\
\hline Interleukin & $\mathbf{r}$ & $\begin{array}{c}\text { correlation } \\
\text { coefficients }\end{array}$ \\
\hline IL-1ra & 0.434 & 0.002 \\
IL-7 & 0.371 & 0.010 \\
IL-8 & 0.321 & 0.028 \\
IL-12 & 0.295 & 0.044 \\
G-CSF & 0.325 & 0.026 \\
IFNg & 0.314 & 0.032 \\
MCP-1 & 0.330 & 0.023 \\
TNFa & 0.402 & 0.005 \\
\hline
\end{tabular}

different between the groups. On the contrary, the other mediators were not affected by the correction.

\section{Discussion}

Although HIV-related deaths have declined dramatically since the introduction of ART, HIV infection is becoming increasingly chronic and people infected with HIV continue to experience raised morbidity and mortality often due to events unrelated to AIDS [19]. In fact, due to the inability of antiretroviral drugs to eradicate the virus from infected reservoir cells, treatment of HIV infection requires permanent systemic therapy. However, even when successfully treated, HIV patients still show higher incidence of age-associated co-morbidities than non-infected individuals. Chronic Immune Activation and Senescence (CIADIS), is a process characterized by a progressive decline of immune system function, usually detected by the expression of cellular or soluble markers derived from innate or adaptive immune responses. Immune activation is associated with progression of HIV disease and increased morbidity and mortality in HIV-infected patients despite ART [20]. In this regard, the validation of a "CIADIS score" based on activation, senescence, and differentiation markers, could help physicians to identify patients at high risk for non-AIDS-related comorbidities [20]. In fact, it is striking evident that cardiovascular diseases are one of the leading non-AIDS causes of death among HIV-positive subjects [21].

Although not fully understood, the probable mechanism involves both chronic inflammation, CD4 cell depletion, endothelial dysfunction and atherosclerosis [22]. It is undoubted that the worst lipid profile (e.g. higher total cholesterol, triglycerides, and LDL-cholesterol than recommended values) that accompanies the HIV affected patients may play a significant role in this [23]. However, in our study the analysis conducted on total cholesterol and triglycerides did not show statistically significant differences between the three groups. This data contrasts with what has been reported in some papers, according to which INI are more "lipid friendly" [24, 25].

Although ART has significantly improved both the quality and lifespan of patients, the life expectancy of treated patients is even shorter than that of uninfected individuals. In particular, while ART may counteract some features of HIV-associated immunosenescence, several anti-HIV drugs may themselves help to amplify other aspects of immune ageing and chronic inflammation [26].

The analysis conducted in the present study reveals that some categories of antiretroviral drugs emphasize the residual inflammation, partly linked to HIV infection itself $[12,13]$.

According to our findings, some inflammatory cytokines of innate immunity (Additional file 1. Figure S1a, c, g) and two pro-inflammatory chemokines (Additional file 1. Figure S1b, f) are more represented in patients taking PI than those receiving INI.

There are several studies comparing the effects of specific antiretrovirals and antiretroviral combinations in ART-naïve individuals initiating their first ART regimen. In one recent ART initiation trial, INI appear to reduce inflammation to a greater degree than NNRTI; however, in the same study, it is not clear if there are beneficial effects on inflammation resulting from treatment with INI compared to PI or between PI and NNRTI [27].

It is plausible that INI may decrease inflammation and immune activation more than other antiretroviral classes, as INI may concentrate at higher levels in enterocytes [28], which is important because HIV infection results in massive depletion of immune cells within the gastrointestinal tract and the resultant microbial translocation may be an important driver of immune activation in HIV. In line with this, we found that INI patients had a tendency to show a lower frequency of extreme cytokine levels when compared to the other groups.

However, in contrast with the above statement, our results show that the IFN- $\gamma$ induced protein 10 (IP-10 or CXCL-10) and eotaxin (also known as $\mathrm{C}-\mathrm{C}$ chemokine

(See figure on next page.)

Fig. 1 Predicted values of cytokines (pg/ml) corrected for covariates (age, sex, smoking status and disease duration) by the ANCOVA approach. Comparisons between groups were performed with the Sidak post-hoc test correction for multiple comparisons. Values of covariates appearing in the model: age $=52.4$ years; sex $=0.67$; smoking status; 0.56 ; HIV duration $=13.9$ years. a IL 1 ra; b IL-8; $\mathbf{c} I L-12 ; \mathbf{d}$ Eotaxin; e IP10; f MIP1b; $\mathbf{g}$ TNF-a. Bars and error bars denote geometric mean and 95\% confidence interval 
a

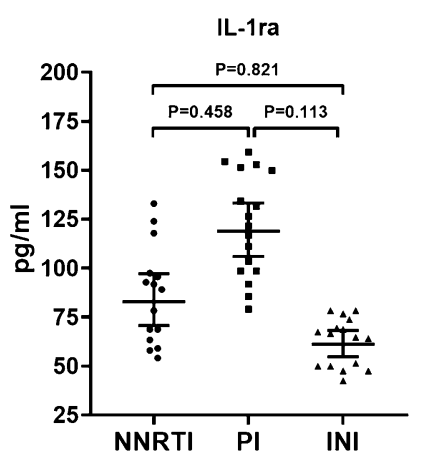

C

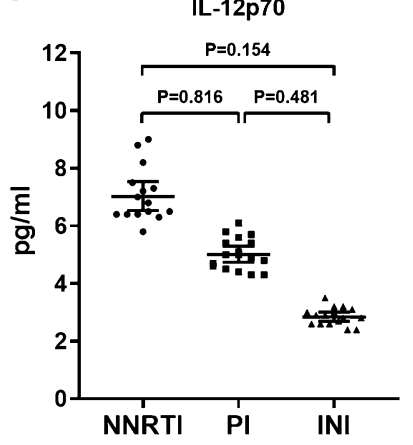

e

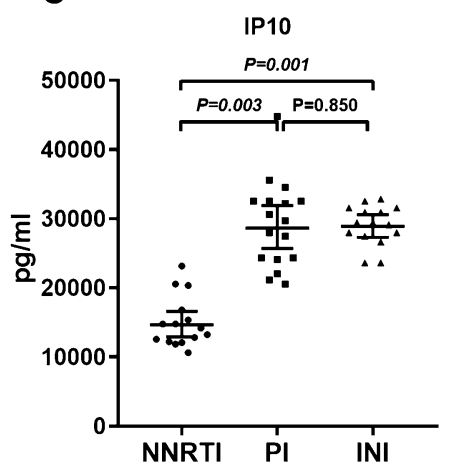

9

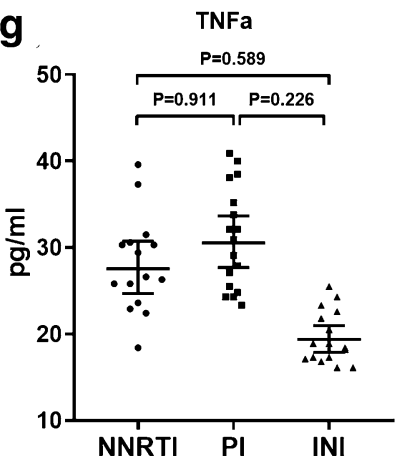

b

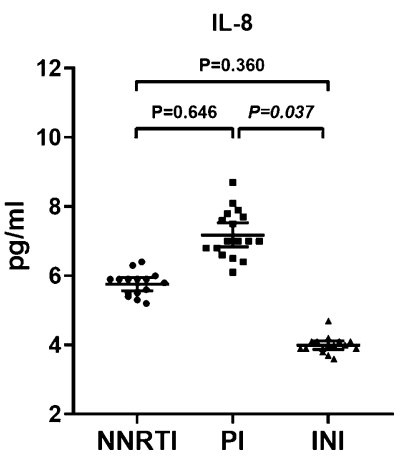

d

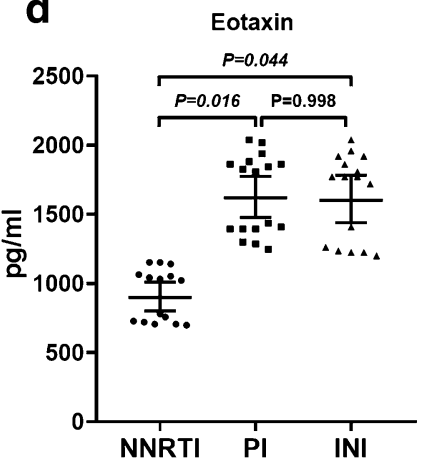

f

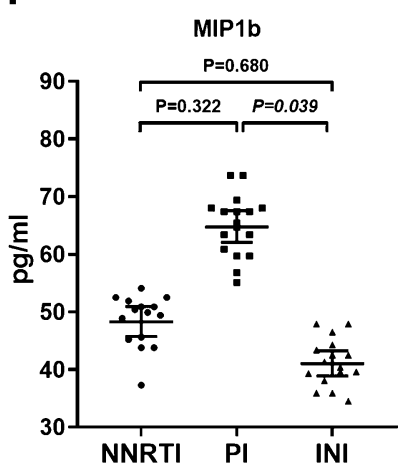


ligand 11, CCL1) are significantly lower in patients treated with NNRTI compared to those belonging to the other groups (Fig. 1e, d). This finding appears relevant especially for IP-10, considering that this mediator is known to be one of the first chemokines to increase following HIV infection [29] and it is involved in immune cell trafficking to inflammatory sites. In particular, the continued production of IFN- $\gamma$ in the lymphoid organs is responsible for a prolonged increase in IP-10 during chronic HIV infection, even in patients taking ART. In fact, IP-10, by increasing the susceptibility of naive $\mathrm{T}$ CD4 + T lymphocytes to HIV infection, improves the production (constitution) of HIV reserves.

The present study is not without limitations, first of all with regard to the small sample size, followed by a cross sectional design and the lack of comparison of the immune mediators tested with other conventional inflammatory or procoagulant markers such as CRP and D-dimer respectively [30]. The lack of a complete set of biomarkers hamper an exhaustive evaluation of the cardiovascular risk and the real inflammatory profile among the three groups and represents the main limitation of the present study.

However, although with limitations, our data seem to highlight the importance of how the choice of the third drug in an antiretroviral regimen can promote the imbalance of inflammation-related immune mediators. In particular, our results seem to discourage the administration of PI as a third drug in a virologically effective antiretroviral regimen, as its use is linked to the detection of higher levels of pro-inflammatory mediators than INI and NNRTI.

Further studies in larger cohorts are needed to confirm the results found, to compare it to conventional markers of inflammation, and to provide their usefulness as a significant clinical tool to help clinicians in choosing a "tailored" antiretroviral regimen.

\section{Supplementary information}

Supplementary information accompanies this paper at https://doi. org/10.1186/s12981-020-00316-w.

Additional file 1: Figure S1. Differences in cytokine concentrations (pg/ $\mathrm{ml}$ ) measured in patients following the therapy simplification regimen. Table S1. Crude and adjusted means of ILs values determined in study population. Table S2. Proportion of outliers found in each group.

\section{Authors' contributions}

Martina Maritati and Carlo Contini equally contributed to the design of the study and the writing of the manuscript; Manola Comar and Tiziana Bellini contributed to the revision of the article and its significant intellectual content; Nunzia Zanotta and Alessandro Trentini contributed to the elaboration of the statistical analysis and interpretation of data; Laura Sighinolfi contributed to the collection of patient's data and the creation of graphics. All authors reviewed, revised and provided final approval of this manuscript before submission.
Funding

The authors received no specific funding for this work.

\section{Availability of data and materials}

The datasets used and/or analysed during the current study are available from the corresponding author on reasonable request.

\section{Ethics approval and consent to participate}

Ethical approval for this study was obtained from the Regional Ethical Committee. The research was carried out in accordance with the ethical principles of the Declaration of Helsinki. Written informed consent was obtained from all subjects before the study.

\section{Consent for publication}

Not applicable.

\section{Competing interests}

The authors declare that they have no competing interests.

\section{Author details}

${ }^{1}$ Section of Infectious Diseases, Department of Medical Sciences, University of Ferrara, 44124 Ferrara, Italy. ${ }^{2}$ Department of Biomedical \& Specialty Surgical Sciences, University of Ferrara, Ferrara, Italy. ${ }^{3}$ Department of Medical Sciences, University of Trieste, Trieste, Italy. ${ }^{4}$ Institute for Maternal and Child Health - IRCCS "Burlo Garofolo", Trieste, Italy. ${ }^{5}$ Infectious Diseases Unit, Azienda Ospedaliero-Universitaria di Cona (Ferrara), Ferrara, Italy.

Received: 17 June 2020 Accepted: 3 September 2020

Published online: 14 October 2020

\section{References}

1. Palella FJ, Delaney KM, Moorman AC, HIV Outpatients Study Investigators, et al. Declining morbidity and mortality among patients with advanced human immunodeficiency virus infection. N Engl J Med. 1998;338(13):853-60.

2. European AIDS Clinical Society Guidelines 2019. Version 10.0. 2019. https ://www.eacsociety.org/files/2019_guidelines-10.0_final.pdf. Accessed Nov 2019.

3. Montaner JSG, Reiss P, Cooper D, For the INCAS Study Group, et al. A randomized, double-blind trial comparing combinations of Nevirapine, Didanosine, and zidovudine for HIV-infected Patients The INCAS trial. JAMA. 1998;279(12):930-7.

4. Friis-Møller N, Sabin CA, Weber R, et al. Combination antiretroviral therapy and the risk of myocardial infarction. N Engl J Med. 2003:349:1993-2003.

5. Crum NF, Riffenburgh RH, Wegner $\mathrm{S}$, et al. Comparisons of causes and death and mortality rates among HIV-infected persons: analysis of the pre-, early and late HAART eras. J Acquir Immune Defic Syndr. 2006:41(2):194-200

6. Carr A, Cooper DA. Advrese effects of antiretroviral therapy. Lancet. 2000;356(9239):1423-30.

7. Guaraldi G, Stentarelli C, Zona S, et al. The natural history of HIV-associated lipodystrophy in the changing scenario of HIV infection. HIV Med. 2014;15(10):587-94

8. Dubé MP, Shen C, Greenwald M, Mather KJ. No impairment of endothelial function or insulin sensitivity with 4 weeks of the HIV protease inhibitors atazanavir or lopinavir-ritonavir in healthy subjects without HIV infection: a placebo-controlled trial. Clin Infect Dis. 2008:47(4):567-74.

9. Kamin D, Hadigan C, Lehrke M, Mazza S, Lazar MA, Grinspoon S. Resistin levels in human immunodeficiency virus-infected patients with lipoatrophy decrease in response to rosiglitazone. J Clin Endocrin Metabol. 2005;90(6):3423-6.

10. Sabin CA, Darminio Monforte A, Friis-Moller $\mathrm{N}$, et al. Changes over time in risk factors for cardiovascular diseases and use of lipid-lowering drugs in HIV infected individuals and impact on myocardial infarction. Clin Infect Dis. 2008. https://doi.org/10.1086/528862.

11. Leite KME, Santos Júnior GG, Godoi ETAM, et al. Inflammatory biomarkers and carotid thickness in hiv infected patients under antiretroviraltherapy, undetectable hiv-1 viral load, and low cardiovascular risk. Arq Bras Cardiol. 2019. https://doi.org/10.5935/abc.20190230. 
12. Phillips AN, Carr A, Neuhaus J, et al. Interruption of antiretroviral therapy and risk of cardiovascular disease in persons with HIV-1 infection: exploratory analyses from the SMART trial. Antivir Ther. 2008;13(2):177-87.

13. Calza L, Manfredi R, Verucchi G. Myocardial infarction risk in HIV-infected patients: epidemiology, pathogenesis and clinical management. AIDS 2010;24(6):789-802.

14. Hazenberg MD, Otto SA, van Benthem $\mathrm{BH}$, et al. Persistent immune activation in HIV-1 infection is associated with progression to AIDS. AIDS. 2003:17:1881-8.

15. Brenchley JM, Price DA, Schacker TW, et al. Microbial translocation is a cause of systemic immune activation in chronic HIV infection. Nat Med. 2006;12:1365-71

16. Wada NI, Jacobson Lp, Margolick JB, et al. The effect of HAART-induced HIV suppression on circulating markers of inflammation and immune activation. AIDS. 2015;29(4):463-71.

17. Comar M, Zanotta N, Bonotti A, et al. Increased levels of C-C chemokine RANTES in asbestos exposed workers and in malignant mesothelioma patients from an hyperendemic area. PLoS ONE. 2014;9(8):e104848.

18. Goff DC Jr, Lloyd-Jones DM, Bennett G, et al. 2013 ACC/AHA guideline on the assessment of cardiovascular risk: a report of the American College of Cardiology/American Heart Association Task Force on Practice Guidelines. Circulation. 2014;129(25 Suppl 2):S49-73.

19. Duffau P, Ozanne A, Bonnet F, et al. Multimorbidity, age-related comorbidities and mortality: association of activation, senescence and inflammation markers in HIV adults. AIDS. 2018;32(12):1651-60.

20. Duffau P, Wittkop L, Lazaro E, ANRS CO3 Aquitaine Cohort Study Group, et al. Association of immune-activation and senescence markers with non-AIDS-defining comorbidities in HIV-suppressed patients. AIDS. 2015;29(16):2099-108.

21. Islam FM, Wu J, Jansson J, et al. Relative risk of cardiovascular disease among people living with HIV: a systematic review and meta analysis. HIV Med. 2012. https://doi.org/10.1111/j.1468-1293.2012.00996.x.

22. Eyawo O, Brockman G, Goldsmith $\mathrm{CH}$, et al. Risk of myocardial infarction among people living with HIV: an updated systematic review and meta-analysis. BMJ Open. 2019;9:e025874. https://doi.org/10.1136/bmjop en-2018-025874.
23. Feeney ER, Mallon PW. HIV and HAART-associated dyslipidemia. TOCMJ. 2011;5:49-63. https://doi.org/10.2174/1874192401105010049.

24. Lennox JL, Landovitz RJ, Ribaudo HJ, Ofotokun I, Na LH, Godfrey C, et al. Efficacy and tolerability of 3 non nucleoside reverse transcriptase inhibitor-sparing antiretroviral regimens for treatment-naive volunteers infected with HIV-1: a randomized, controlled equivalence trial. Ann InternMed. 2014;161(7):461-71.

25. Martinez E, Larrousse M, Llibre JM, Gutierrez F, Saumoy M, Antela A, et al. Substitution of raltegravir for ritonavir-boosted protease inhibitors in HIVinfected patients: the SPIRAL study. AIDS. 2010;24(11):1697-707.

26. Franzese $\mathrm{O}$, Barbaccia ML, Bonmassar E, Graziani G. Beneficial and detrimental effects of antiretroviral therapy on HIV-associated immunosenescence. Chemotherapy. 2018;63(2):64-75.

27. Hileman CO, Funderburg NT. Inflammation, immune activation, and antiretroviral therapy in HIV. Curr HIV/AIDS Rep. 2017. https://doi. org/10.1007/s11904-017-0356-x.

28. Patterson KB, Prince HA, Stevens T, Shaheen NJ, Dellon ES, Madanick RD, et al. Differential penetration of raltegravir throughout gastrointestinal tissue: implications for eradication and cure. AIDS. 2013;27(9):1413-9.

29. Jie L, Xiaowan $Y$, Hong S, Yongjun J. IP-10 is highly involved in HIV infection. Cytokine. 2019;115:97-103.

30. Pinzone R, Di Rosa M, Cacopardo B, Giuseppe NG. HIV RNA suppression and immune restoration: can we do better? Clin Dev Immunol. 2012;2012:515962.

\section{Publisher's Note}

Springer Nature remains neutral with regard to jurisdictional claims in published maps and institutional affiliations.
Ready to submit your research? Choose BMC and benefit from:

- fast, convenient online submission

- thorough peer review by experienced researchers in your field

- rapid publication on acceptance

- support for research data, including large and complex data types

- gold Open Access which fosters wider collaboration and increased citations

- maximum visibility for your research: over 100M website views per year

At BMC, research is always in progress.

Learn more biomedcentral.com/submissions 